\title{
Gewährleistung des Datenschutzes - externe und professionelle Kodierrevision macht es möglich
}

Gabriela Langa,

Hanspeter Kuhn ${ }^{b}$,

Beatrix Meyer,

Petra Ingenpass ,

Judith Wagnere

a Rechtsanwältin, FMH-Rechtsdienst

b Fürsprecher stv. Generalsekretär

c Leiterin Tarifdienst FMH und Bereich SwissDRG

d Dr. med., stv. Leiterin Bereich SwissDRG der FMH

e Dr. sc. hum, Leiterin eHealth der FMH

1 AB 2007 N 442-446.

2 Vgl. Kaufmann S. Stand der Einführung des DRG-Systems in der Schweiz - aus Sicht der Krankenversicherer. In: Gesundheitssysteme im Wandel, Rebscher H Kaufmann S (Hrsg.). Economica. 2009; S. 256 N 11. Und: Datenschutz unter DRG aus Sicht der santésuisse. Referat von Wolfram Strüwe, Helsana AG, Zürich, anlässlich der Frühlingstagung 2010 der SGMC vom 27. April 2010, Bern.

3 Website der CSS, zuletzt konsultiert 9. August 2010.

Korrespondenz: Rechtsdienst FMH Postfach 170 CH-3000 Bern 15 Tel. 0313591111 Fax 0313591112 lex@fmh.ch
$\mathrm{Ab}$ 1. Januar 2012 werden in der ganzen Schweiz akutstationäre Behandlungen mit diagnosebezogenen Fallpauschalen (DRG) abgegolten. Kantone und Versicherer übernehmen dabei die Vergütung anteilsmässig, wobei der kantonale Anteil mindestens $55 \%$ beträgt.

Gemäss Krankenversicherungsgesetz (KVG) muss der Leistungserbringer dem Schuldner eine detaillierte und verständliche Rechnung zustellen. Er muss ihm auch alle Angaben machen, die dieser benötigt, um die Berechnung der Vergütung und die Wirtschaftlichkeit der Leistung überprüfen zu können (Art. 42 Abs. 3 KVG).

Doch welche Angaben sind dafür notwendig? Diese Frage gibt unter den Partnern im Gesundheitswesen immer wieder Anlass zu kontroversen Diskussionen. Die ärztliche Schweigepflicht und die Datenschutzinteressen der Patienten/Versicherten kollidieren mit den Kontrollinteressen der Versicherer.

\section{Bearbeitung von Personendaten - was gilt?}

Wichtig zu wissen ist, dass für das Bearbeiten von Personendaten das im Bundesgesetz über den Datenschutz (DSG) verankerte Verhältnismässigkeitsprinzip (Art. 4 Abs. 2 DSG) gilt: Nur diejenigen Daten dürfen bearbeitet werden, die für die konkrete Aufgabenerfüllung geeignet und notwendig sind - und es darf kein Instrument vorhanden sein, das mit einem weniger weitgehenden Eingriff zum gleichen Ziel führt (Prinzip des geringstmöglichen Eingriffs).

Richtigerweise hat der Gesetzgeber im Zusammenhang mit der neuen Spitalfinanzierung eine Schlüsselstelle im KVG nicht geändert. In Art. 42 Abs. 4 KVG steht auch in Zukunft «Der Versicherer kann eine genaue Diagnose oder zusätzliche Auskünfte medizinischer Natur verlangen.»

Vor der Abstimmung zurückgezogen wurde hingegen der Antrag Humbel Näf, der in einem neuen Abs. $4^{\text {bis }}$ vorgesehen hätte: «Bei leistungsbezogenen Fallpauschalen muss der Leistungserbringer auf der Rechnung alle Angaben machen, welche für die Ermittlung der korrekten Fallpauschalen notwendig sind, insbesondere auch die relevanten Diagnosen und Prozeduren» [1].

Somit war es gerade nicht die Meinung des Gesetzgebers, für alle stationären Spitalpatienten routine- mässig die genauen Diagnosen und Prozeduren dem Versicherer bekanntzugeben. Es gilt auch in Zukunft das Stufenprinzip, wie es der Datenschutzbeauftragte seit Jahren postuliert: Wenn nach der Rechnungsstellung Fragen offen bleiben, kann der Versicherer Rückfragen stellen.

\section{Versicherer verlangen alle Angaben, die für die Gruppierung des Falles notwendig sind} Die Versicherer vertreten den Standpunkt, sie müssten personenbezogen - und damit weder anonymisiert noch pseudonymisiert - alle Angaben erhalten, die für die Gruppierung des Falles notwendig sind. Unter dem verharmlosenden Begriff «Minimum Data Set» (MDS) wollen sie unter anderem sämtliche Hauptund Nebendiagnosen sowie alle Behandlungen eines Patienten in der Form von Codes [2]. Auf der Website eines grossen Versicherers tönt das so: «Beim MDS handelt es sich um einen klar definierten Datensatz, der auch vom BfS (Bundesamt für Statistik) eingefordert wird. Dem Spital entsteht somit kein zusätzlicher Aufwand. Die Krankenversicherer beabsichtigen nicht, die Nachcodierung im Einzelfall vorzunehmen, sondern ermitteln mittels statistischer Verfahren Auffälligkeiten, die dann gezielt genauer untersucht werden können» [3].

Zur Erinnerung: Das Bundesamt für Statistik erhebt diese Daten nicht personenbezogen, sondern anonymisiert. Und wenn die Forderung, sozusagen einfachheitshalber den BfS-Datensatz auch dem Versicherer zuzustellen, zum Nennwert zu nehmen ist, so sind darüber hinaus noch Daten betroffen, die für die DRG-Gruppierung gar nicht relevant sind.

\section{Kantone mit DRG konzentrieren sich auf externe professionelle Kodierrevision}

Die Kantone, in denen schon heute mit diagnosebezogenen Fallpauschalen abgerechnet wird, konzentrieren sich auf die externe professionelle Kodierrevision. Kein Kanton postuliert, er müsste zur Kontrolle seines Beitrags an die Spitalbehandlungskosten personenbezogene Daten wie das von den Kassen postulierte Minimum Data Set erhalten [4]. Der Vertreter der GDK weist im Hinblick auf die Einführung von SwissDRG auf die zentrale Bedeutung der Kodierrevision hin [5]. 
4 Es war ein Mitarbeiter einer externen Kodierrevisionsstelle, der an der Frühlingstagung 2010 der SGMC vom 27. April 2010 in Bern auf die bemerkenswerte Situation hingewiesen hat, dass die Kantone, die den grösseren Teil der Spitalkosten finanzieren, sich auf die externe, unabhängige Kodierrevision als das geeignete Kontrollinstrument konzentrieren, und nur der «Juniorpartner» Krankenversichere detaillierte personenbezogenes Daten erhalten möchte. Honni soit qui mal y pense.

5 Vgl. GDK-Vizepräsident Carlo Conti in GDK santé Mai 2009: «Die Abrechnungsregeln enthalten eine Reihe von Massnahmen, welche die Abgeltung bei Wiedereintritten ins Spital, bei Verlegung oder Rückverlegung regeln. Sie wirken so einer künstlichen Fallgenerierung entgegen und garantieren, dass Patienten solange wie notwendig behandelt werden. Eine einheitliche Kodierkontrolle stellt die korrekte Leistungserfassung sicher, und eine statistische Auswertung von Struktur- und Leistungsdaten als Monitoring deckt unerwünschte Auswirkungen auf.»

6 Lang, Miotti. Rechtsgutachten vom 28. März 2008 zur Weitergabe von Patientendaten an die Versicherer zuhanden von $\mathrm{H}+$ die Spitäler der Schweiz. Auszug aus Schlussfolgerung. S. $75 \mathrm{f}$

7 Verordnung vom 27. Juni 1995 über die Krankenversicherung (KVV), Änderungen per

1. Januar 2009, Änderungen und Kommentar im Wortlaut.

8 VV-Revision, a.a.O, S. 9.

9 AB 2007 N 445.

\section{H+: externe professionelle Kodierrevision statt Minimal Data Set}

$\mathrm{H}+$ hat ein Rechtsgutachten in Auftrag gegeben, das am 28. März 2008 veröffentlicht wurde. Die Gutachter Elisabeth Lang und Roland Miotti kommen zum Schluss, dass es bei den DRG um die «generelle Überprüfung der Leistungserbringung nach qualitativen und quantitativen wirtschaftlichen Kriterien, einer Wirtschaftlichkeitskontrolle mittels Durchschnittskostenvergleich» geht.

Im Gegenzug gilt gemäss Gutachter: Die «systematische Datenweitergabe nach Art. 42 Abs. 3 KVG (ist) jedoch nicht» für «vertiefte individualorientierte Informationen im Einzelfall» geschaffen. «Vertiefte Einzelfallinformationen - wozu auch das Minimal Data Set zu zählen sein dürfte - haben sich samt den dazugehörigen Modalitäten auf Art. 42 Abs. 4 KVG zu stützen. Folgt man dieser Einschätzung, so würden mit der Weitergabe des Minimal Data Sets strafrechtliche und datenschutzrechtliche Fehler (Verletzung des Patientengeheimnisses und des informationellen Selbstbestimmungsrechts) begangen» [6]

\section{Bundesrat: Versicherer braucht personen- bezogene Daten, Aufbewahrung aber pseudonymisiert}

Im Kommentar zur KVV-Revision per 2009 [7] steht im allgemeinen Teil auf Seite 4 zu Rechnungskontrolle und Datenbereitstellung für die Weiterentwicklung des Tarifsystems: «Die Einführung von leistungsbezogenen Pauschalen in der Form diagnosebezogener Fallpauschalen macht Datenlieferungen vom Leistungserbringer an den Versicherer erforderlich. (...) Die Datenweitergabe an die Versicherer ist unabdingbar, weil diese zur Rechnungskontrolle personenbezogene Daten benötigen. Zudem werden die Daten zur Weiterentwicklung des Tarifsystems und zur Kontrolle der Systemumsetzung durch die mit dieser Aufgabe betrauten Stellen benötigt; letzteres aber in pseudonymisierter Form und nicht personenbezogen.» gnosen- und Prozedurenbekanntgabe mit der Rechnung erwähnt. Bundesrat Couchepin hatte dazu schon in der Spitalfinanzierungsdebatte im Nationalrat ausgeführt: «... la transmission de diagnostics généraux nécessaires pour le traitement administratif de cas ordinaires est permise, mais des indications plus détaillées peuvent être exigées le cas échéant ultérieurement» [9].

\section{Bundesverwaltungsgericht verlangt flankierende Massnahmen}

Grundlage des Urteils des Bundesverwaltungsgerichts vom 29. Mai 2009 (C 6570/2007) war ein Tarifvertrag zwischen santésuisse und den öffentlichen Spitälern Bern, worin u. a. die systematische Weitergabe der Diagnosen mit der Eintrittsmeldung resp. der Diagnosenund Eingriffscodes mit der Rechnung vereinbart wurden. Der Regierungsrat des Kantons Bern hatte seine Zustimmung zu diesen zwei Punkten verweigert, den Tarifvertrag ansonsten aber genehmigt. Aufgrund der durch santésuisse erhobenen Beschwerde hatte das Bundesverwaltungsgericht zu entscheiden, ob der Regierungsrat des Kantons Bern seine Zustimmung zu Recht verweigert hatte.

Entgegen der Auffassung des Regierungsrates kam es in seinem Urteil zum Schluss, dass das Krankenversicherungsgesetz eine genügende Rechtsgrundlage für eine tarifvertraglich vereinbarte systematische Weitergabe von Diagnosen- und Eingriffscodes bilde. Eine solche Vereinbarung sei nach Ansicht des Gerichts aber nur dann zulässig, wenn deren genaue Ausgestaltung gemäss dem Prinzip des geringstmöglichen Eingriffs von den Parteien geregelt werde. Entsprechend fehlten im zu beurteilenden Tarifvertrag u.a. Regeln bezüglich der genauen Ausgestaltung der Datenweitergabe. Daten über «heikle», «beziehungsweise von einem (erheblichen) Teil der Bevölkerung als stigmatisierend empfundene Krankheiten» sollen nach Ansicht des Gerichts nicht in standardisierter Form übermittelt werden, sondern nur an den Vertrauensarzt

\section{Externe professionelle Kodierrevision ist das geeignete Instrument für eine angemessene Kontrolle der Codierung als Grundlage von Rechnungen}

Im besonderen Teil ist zur Rechnungsstellung auch zu lesen: «Weil die Aufbewahrung der Daten (im Datenschutzgesetz; Anm. d. Autoren) nicht geregelt ist, wird in Absatz $1^{\text {ter }}$ festgehalten dass die Daten in pseudonymisierter Form aufzubewahren sind und dass die Pseudonymisierung nur durch den Vertrauensarzt des Versicherers aufgehoben werden kann» [8].

Klar ist, dass die Rechnung personenbezogen sein muss. Klar ist hingegen auch, dass der Bundesrat mit keinem Wort die systematische und detaillierte Dia- weitergeleitet werden dürfen. Im Vertrag seien zudem die notwendigen Präzisierungen im Sinne des Verhältnismässigkeitprinzips und des Datenschutzes vorzunehmen. Namentlich seien die Informationen zu definieren, die ausschliesslich an den Vertrauensarzt gehen. Weiter seien die Patienten ausdrücklich auf ihr Recht hinzuweisen, dass sie die Weitergabe der medizinischen Angaben an den Vertrauensarzt oder die Vertrauensärztin verlangen können. Das Bundesverwaltungsgericht kam zum Schluss, dass der Regierungsrat 
die Genehmigung zu Recht verweigert hatte, jedoch mit falscher Begründung.

Nicht diskutiert hat das Bundesverwaltungsgericht die Möglichkeit einer externen professionellen Kodierrevision und die Bedeutung dieses Instruments im Hinblick auf die Verhältnismässigkeit systematischer personenbezogener Datenweitergaben an den Versicherer.

\section{Bernischer Datenschutzbeauftragter zur Umsetzung des Urteils von 2009}

Der Datenschutzbeauftragte des Kantons Bern hat am 30. April 2010 in seinem Bericht an den Regierungsrat unter anderem festgehalten:

«Das Urteil geht aus datenschutzrechtlicher Sicht zu wenig differenziert auf die komplexe Materie ein. (...) $\mathrm{Zu}$ beachten ist zudem, dass das Bundesverwal- tungsgericht in seinem Entscheid von der herkömmlichen, leistungsbezogenen Abrechnung und nicht vom Modell der Abrechnung auf Grund von Fallpauschalen ausgegangen ist. Daraus folgt, dass die im Urteil gemachten Ausführungen nicht ohne weiteres auch für den hier zugrunde liegenden FallpauschalenTarifvertrag anwendbar sind. (...) Die Krankenkassen legen das Urteil - wohl auch aufgrund des Tarifstrukturvertrags, welcher in Ziffer 8.1 Abs. 2 die Übermittlung der Codes in vollständiger Länge vorsieht - so aus, als ob der Datentransfer des ganzen Codes stets gerechtfertigt ist. Das Bundesverwaltungsgericht äusserte sich im Urteil jedoch nicht konkret zur Übermittlung der vollständigen Codes. Es hielt lediglich fest, dass je detaillierter ein Code übermittelt wird, desto mehr ‘flankierende Massnahmen〉 greifen müssen.»

\section{Die Position der FMH}

\section{Externe professionelle Kodierrevision als das zentrale Kontrollinstrument}

Es ist unbestritten, dass eine angemessene Kontrolle der Codierung als Grundlage für die Rechnungen nötig ist. Diese Überprüfung kann nur gestützt auf die Krankengeschichte (Patientenakte) erfolgen. Dafür ist die externe professionelle Kodierrevision das geeignete Instrument. «Extern», weil sie durch einen unabhängigen Revisor durchgeführt wird, «professionell», weil die Revisoren dafür qualifiziert sind.

Die externe professionelle Kodierrevision «vergleicht die Patientendossiers mit der Kodierung. Sie setzt somit dort an, wo Fehler passieren und erkannt werden können. Die Revision erfolgt systematisch durch aussagekräftige und regelmässige Stichproben» [10]. Die Prüfkriterien sind durch ein national einheitliches Reglement definiert. Hierzu wird in Art. 59d Abs. 2 KVV präzisiert: «Im Falle eines auf einem Patienten-Klassifikationssystem vom Typus DRG basierenden leistungsbezogenen Vergütungsmodells muss der Tarifvertrag zusätzlich das Kodierungshandbuch sowie ein Konzept zur Kodierrevision enthalten.» «Der Datenschutz (bei der externen professionellen Kodierrevision; Anm. d. Autoren) ist optimal, die Effizienz hoch. Die Versicherer müssen nicht alle für sich Kontrollmechanismen aufbauen und alle Spitäler werden gleich behandelt» [11].

Die Revision findet einmal pro Jahr im zu revidierenden Spital statt. Der Revisor wird gemeinsam von den Vertragsparteien bestimmt, wobei die Spitäler ein Vorschlagsrecht haben. Können sich die Vertragsparteien nicht einigen, wird der Revisor hoheitlich vom Kanton bestimmt. Die Revision wird anhand von Stichproben durchgeführt, die der Revisor in Zusammenarbeit mit dem Spital aus allen nach SwissDRG fakturierten Fällen des Berichtsjahres nach dem Zufallsprinzip zieht. Für die erste Revision nach SwissDRG wird die Stichprobengrösse auf 180 Fälle bei nicht-universitären Spitälern festgelegt. Für Universitätsspitäler beträgt der Stichprobenumfang 300 Fälle. Der Revisor kodiert jeden Fall der Stichprobe anhand der vollständigen Krankengeschichte einzelfallbezogen nach, d.h. die Ursprungs-

\section{Das «Minimum Data Set» müsste richtigerweise «Maximum Daten Friedhof» genannt werden}

kodierung ist bekannt. Und der Revisor überprüft ebenfalls, ob die ursprüngliche Kodierung des Spitals mit der Rechnungsstellung kongruent ist. Über das Ergebnis der Revision hat der Revisor einen Bericht zu erstellen. Aus dem Inhalt des Berichts dürfen keine Rückschlüsse auf die Identität von Patienten möglich sein» [12].

Die Kodierrevision ist keine Taube auf dem Dach, sondern Realität in vielen Kantonen, die heute mit diagnosebezogenen Pauschalen abrechnen. Und die Kodierrevision wird es auch unter SwissDRG geben, denn so haben es die Tarifpartner vereinbart» [13].

\section{Statistische Auswertungen auf Basis anonymisierter Daten}

Der Zweck der DRG besteht u.a. darin, möglichst aussagekräftige Durchschnittskostenvergleiche zu ermöglichen. Diese werden mit anonymisierten Daten durchgeführt. Dies haben die Gutachter Lang und Miotti im 2008 richtig dargelegt. 
14 Hier wird der wissenschaftlich übliche Begriff Minimal Data Set verwendet. Die Krankenversicherer sprechen in letzter Zeit von Minimum Data Set.

15 Grouper: «Die Zuweisung einer Hospitalisierung zu einer bestimmten DRG erfolgt über eine Gruppierungssoftware, den so genannten «Grouper». Der Klassifizierungsalgorithmus basiert auf den medizinischadministrativen Falldaten.» (vgl. zu den Begriffen die Website von SwissDRG: www.swissdrg.org/de/ 07 casemix office/ Wichtige_Begriffe. asp? navid=16)

16 Hölzer S Bienlein M. $\mathrm{H}+$ Bundeshaus; September 2007. S. 3

17 Vgl. Kaufmann S. Stand der Einführung des DRGSystems in der Schweiz aus Sicht der Krankenversicherer. In: Gesundheitssysteme im Wandel, Rebscher H, Kaufmann S (Hrsg.). Economica. 2009; S. $256 \mathrm{~N} 12$.

18 Website der CSS, zuletzt konsultiert 9. August 2010.

\section{Vertrauensarzt für Einzelfallprüfung}

Der Vertrauensarzt erfüllt im Zusammenhang mit der Einzelfallprüfung als sachkundiger Berater und «Filter» eine wichtige Rolle für den Krankenversicherer.

\section{Minimal Data Set ist ungeeignet und unverhältnismässig}

Mit dem Minimal Data Set [14] kann der Versicherer nur prüfen, «ob das Berechnungsprogramm von SwissDRG, der sogenannte Grouper [15], richtig funktioniert. Dies sollte der Fall sein, da der Grouper von der SwissDRG AG bereitgestellt wird. Kodierung, Wirtschaftlichkeit und Angemessenheit sind auf diese Weise jedoch nicht überprüfbar und der Datenschutz bleibt auf der Strecke» [16]. Dies hat H+ schon 2007 richtig dargelegt. Wenn die Versicherer die Gruppierung durch die Software überprüfen möchten, ist dafür die Zertifizierung der Grouper-Software ein geeigneter Weg, nicht aber, dieselben Daten mehrfach durch die Software laufen zu lassen.

Den Versicherern ist bewusst, dass die Mehrheit der Rechnungen korrekt gestellt wird und dass zwischen Aufwand und Nutzen einer systematischen Detailprüfung kein vernünftiges Verhältnis bestehen würde. Sie geben an, sie wollten mit dem Minimum Data Set die inkorrekten von den korrekten Rechnungen trennen können [17]. Wenn die Versicherer «mittels statistischer Verfahren Auffälligkeiten ermitteln» wollen, «die dann gezielt genauer untersucht werden können» [18], wäre es geradezu ein Lehrbuchbeispiel des unzulässigen Datensammelns auf Vorrat, wenn sie dafür mit jeder Rechnung alle Haupt- und Nebendiagnosen und alle Prozeduren personenbezogen detailliert erhielten. Das «Minimum Data Set» müsste richtigerweise «Maximum Daten Friedhof» genannt werden. Dazu darf es nicht kommen. (Oder möchten die Versicherer all die Daten vielleicht deshalb erhalten, um vermehrt Risikoselektion betreiben zu können?)

\section{Liste heikler Diagnosen oder geringerer Detaillierungsgrad sind nicht praktikable Lösungen}

Das Bundesverwaltungsgericht und der Datenschutzbeauftragte des Kantons Bern fordern zu Recht Ein- schränkungen in der Datenübermittlung von Diagnosen und Prozeduren an die Krankenversicherer. Allerdings sind die von ihnen vorgeschlagenen Lösungsansätze nicht praktikabel:

- Es ist praktisch nicht möglich, eine Liste mit sogenannten stigmatisierenden bzw. heiklen Diagnosen zu erstellen. Was eine heikle Information ist, hängt zentral von Wertvorstellungen und der konkreten sozialen Situation des Patienten ab. Dies hat der Gesetzgeber im Kanton Genf richtig erkannt, der dem einzelnen Patienten das Recht gibt, Teile seiner Patientendaten als stigmatisierend zu kennzeichnen und damit den Zugriff von Behandelnden auf diese Daten einzuschränken.

- Es macht im DRG-System keinen Sinn, Diagnosen oder Prozeduren aus dem Minimal Data Set zu entfernen oder mit einem geringen Detaillierungsgrad darzustellen, weil die Gruppierung in diesem Fall nicht mehr nachvollzogen werden könnte. Damit wäre die Rückfrage des Krankenversicherers vorprogrammiert.

Weil das Bundesverwaltungsgericht in seinem Urteil das zentrale Instrument der externen professionellen Kodierrevision nicht diskutiert, hinkt das Urteil der in vielen Kantonen praktizierten Realität hinterher und ist als Leitentscheid nicht geeignet.

\section{Fazit}

Sachgerecht ist die Kombination von externer professioneller Kodierrevision mit Durchschnittskostenvergleichen, welche die Versicherer, gestützt auf anonymisierte Daten, durchführen.

Ungeignet, nicht erforderlich, unverhältnismässig und vom Gesetzgeber nicht vorgesehen wäre hingegen, dem Versicherer personenbezogen mit jeder Rechnung detaillierte Diagnosen und Prozeduren mitzuteilen. 